\title{
Online Interactive Experiments on Networks
}

\author{
Mohsen Mosleh \\ Sloan School of Management \\ Massachusetts Institute of Technology \\ Cambridge, MA, USA \\ mmosleh@mit.edu
}

\section{TUTORIAL ABSTRACT}

Conducting human experiments using crowdsourcing platforms, such as Amazon Mechanical Turk, has made it possible to collect a much larger amount of experimental data in a much shorter period of time relative to what was possible in traditional physical lab settings. This has provided a new suite of methods for conducting randomized experiments in socio-technical systems, allowing for straightforward causal inference [1-4]. However, using crowdsourcing platforms to experimentally study real-time interactions between individuals presents numerous practical challenges. These studies need fairly large groups of subjects to be present simultaneously in each session, and outcomes typically occur at the level of the group (i.e., session) rather than the individual. Yet most crowdsourcing platforms are not designed to facilitate simultaneous structured interactions between subjects. Thus, it can be difficult (and expensive) to recruit enough participants to achieve a sufficient degree of statistical power (especially for session-level outcomes).

In this tutorial, we will discuss best practices for designing and conducting online social network experiments where human subjects (and programmed bots) interact simultaneously within a specified network structure. We will show how the experimental design can be informed by computational models in an iterative process (i.e., using experimental data to calibrate the computational model and use the computational model to optimize the design and find the right parameters for the experiments). We will also introduce additional tools/platforms that facilitate conducting such studies and walk the audience through the implementation steps of a typical experiment on networks using customized and publicly available software.

\section{CCS CONCEPTS}

- Human-centered computing $\rightarrow$ Human computer interaction (HCI); Collaborative and social computing; • Information systems $\rightarrow$ Information systems applications; World Wide Web; •Applied computing $\rightarrow$ Law, social and behavioral sciences;

\section{KEYWORDS}

Social Networks; Online Experiments; Crowdsourcing; Amazon Mechanical Turk

\section{TUTORIAL CONTENT}

The tutorial content and material can be accessed here: http://mohsenmosleh.com/websci-tutorial/

\section{REFERENCES}

[1] Brackbill, and Damon Centola. "Network dynamics of social influence in the wisdom of crowds." Proceedings of the national academy of sciences (2017): 201615978.

[2] Shirado, Hirokazu, and Nicholas A. Christakis. "Locally noisy autonomous agents improve global human coordination in network experiments." Nature 545.7654 (2017): 370.

[3] Nishi, Akihiro, et al. "Inequality and visibility of wealth in experimental social networks." Nature 526.7573 (2015): 426.

[4] Rand, David G., Samuel Arbesman, and Nicholas A. Christakis. "Dynamic social networks promote cooperation in experiments with humans." Proceedings of the National Academy of Sciences 108.48 (2011): 19193-19198.

\footnotetext{
Permission to make digital or hard copies of part or all of this work for personal or classroom use is granted without fee provided that copies are not made or distributed for profit or commercial advantage and that copies bear this notice and the full citation on the first page. Copyrights for third-party components of this work must be honored. For all other uses, contact the Owner/Author.

WebSci '19 Companion, fune 30-fuly 3, 2019, Boston, MA, USA

(C) 2019 Copyright is held by the owner/author(s).

ACM ISBN 978-1-4503-6174-3/19/06.

https://doi.org/10.1145/3328413.3329795
} 